Voix et Images

volxetimages

\title{
Le quotidien en mode mineur : Le bruit des choses vivantes d'Élise Turcotte
}

\author{
Daily Life in a Minor Key: Le bruit des choses vivantes by Élise \\ Turcotte
}

\section{Lo cotidiano en modo menor: Le bruit des choses vivantes, de Élise Turcotte}

\section{Marie-Pascale Huglo}

Volume 34, numéro 3 (102), printemps-été 2009

Trajectoires de l'auteur dans le théâtre contemporain

URI : https://id.erudit.org/iderudit/037667ar

DOI : https://doi.org/10.7202/037667ar

Aller au sommaire du numéro

Éditeur(s)

Université du Québec à Montréal

ISSN

0318-9201 (imprimé)

1705-933X (numérique)

Découvrir la revue

Citer cet article

Huglo, M.-P. (2009). Le quotidien en mode mineur : Le bruit des choses vivantes d'Élise Turcotte. Voix et Images, 34(3), 99-115. https://doi.org/10.7202/037667ar
Résumé de l'article

Cet article vise à analyser la place et la fonction du quotidien dans le roman minimaliste d'Élise Turcotte, Le bruit des choses vivantes. Plutôt que de considérer le quotidien comme un objet privilégié de la fiction intimiste, on examinera la façon dont le quotidien est construit et valorisé dans le roman. Réciproquement, on verra comment ce roman s'élabore narrativement à partir du quotidien. L'enjeu est donc de revenir sur le rapport peut-être trop évident que l'on tend à établir entre le quotidien, la fiction intimiste et le minimalisme. Tout en cherchant à mieux cerner les enjeux narratifs du quotidien, on tentera de dégager ce qui définit le « mode mineur " dans ce roman. 


\title{
LE QUOTIDIEN EN MODE MINEUR : LE BRUIT DES CHOSES VIVANTES D'ÉLISE TURCOTTE 1
}

$+++$

\author{
MARIE-PASCALE HUGLO \\ Université de Montréal
}

Le premier roman d'Élise Turcotte raconte, à travers les mille et une petites choses du quotidien, une année dans la vie d'Albanie et de sa fille Maria. Relié à l'histoire collective par le biais des images télévisuelles, le plus souvent catastrophiques, qui «se gravent dans la tête» d'Albanie, Le bruit des choses vivantes ${ }^{2}$ présente un portrait de femme ancré dans la société contemporaine. Mère monoparentale d'une fille de trois ans, trentenaire, bibliothécaire peu assidue acceptant volontiers de faire des virées avec Jeanne - séparée elle aussi et mère d'un petit Gabriel -, Albanie est un personnage féminin caractéristique de la "réalité d'aujourd'hui», qui reconnaît dans une des filles du feuilleton télévisé Thirtysomething un double d'elle-même. Les nombreux renvois à la culture télévisuelle tissent la toile de fond d'une américanité résolument contemporaine. Pourtant, aussi enraciné soit-il dans le Québec d'aujourd'hui, ce roman s'attache moins à décrire une réalité qu'à montrer comment cette réalité est perçue de l'intérieur. C'est en effet sous la forme d'une introspection que le récit se déploie, les événements petits et grands étant tous perçus et médiatisés par Albanie.

Le point de vue subjectif et intériorisé du roman a été reçu par la critique comme la marque d'un intérêt renouvelé pour l'intime et le minimalisme ${ }^{3}$. Pour Lori

$$
+++
$$

1 Cet article s'inscrit dans le cadre d'une recherche subventionnée par le CRSH sur la mise en récit du quotidien dans la littérature contemporaine. 2 Élise Turcotte, Le bruit des choses vivantes, Montréal/Arles, Leméac/Acte sud, coll. «Babel», 1998 [1991], 244 p. Désormais, les références à ce roman seront indiquées par le sigle $B C V$, suivi du folio, et placées entre parenthèses dans le texte. 3 L'intimisme est considéré comme l'une des caractéristiques de la littérature contemporaine au Québec. L'intérêt pour le quotidien et l'intériorité participent de ce courant associé, dans le contexte québécois, au minimalisme, qui n'est cependant pas homogène ni exclusif. (Voir « Jacques Poulin et le roman en mode mineur» et «La poésie et la fiction intimiste», dans Michel Biron, François Dumont et Élisabeth Nardout-Lafarge, Histoire de la littérature québécoise, Montréal, Boréal, 2007, p. 544-551 et p. 604-612 respectivement). En France, le lien entre le quotidien et l'intime est moins souligné par la critique des fictions minimalistes: celles-ci vont des jeux parodiques qui enlisent le roman dans les trivialités du quotidien, à la manière de Jean-Philippe Toussaint, jusqu'à la recharge de sens et de valeur accordée aux petites choses de la vie dont les proses de Philippe Delerm sont représentatives. Voir Warren Motte, Small Worlds. Minimalism in Contemporary French Literature, Lincoln, University of Nebraska Press, 1999, 212 p.; Jacques Poirier, «Le pas grand-chose et le presque rien", Bruno Blanckeman, Alice Mura-Brunel et Marc Dambre (dir.), Le roman français au tournant du XXI ${ }^{e}$ siècle, Paris, Presses Sorbonne nouvelle, 2004, p. 371-380. 
Saint-Martin, ce renouvellement est associé au métaféminisme qui implique, entre autres, une métamorphose de la maternité et le souci pour le quotidien dans l'écriture des femmes ${ }^{4}$. Cette nouvelle subjectivité se définit plutôt, selon Pierre Nepveu, en termes de ritualisation qui, après la fondation et la transgression caractéristiques de la littérature québécoise d'avant les années 1980, propose une manière personnelle et intime d'habiter le monde ${ }^{5}$. Cette nouvelle manière se distinguerait tant du "désir d'action et de puissance» typiquement romanesque que des jeux postmodernes; elle traduirait la quête subjective d'un chez-soi ouvert au monde mais établi dans «la maison et la vie quotidienne ${ }^{6}$ ». Qu'on l'associe au féminin ou pas, l'écriture d'Élise Turcotte participe d'un nouvel art romanesque «tout intérieur, sensationnel et subjectif», laissant derrière lui, écrit Andrea Oberhuber, "des considérations politiques explicites et théoriques», opposant "au chaos du monde contemporain» «l'intimité peu bruyante» et «la subjectivité souvent fragile et rêveuse, révélant sur le plan des thèmes aussi bien que sur le plan esthétique une modestie qui cherche à "aménager le quotidien et à y créer des points de résistance, des rituels d'habitation ou d'évasion" plutôt qu'à refaire le monde ${ }^{7}$ ». Oberhuber s'accorde en cela avec Michel Biron, pour qui les fictions d'Élise Turcotte sont un exemple "d'écritures délicates et retenues, incompatibles avec le roman à grand déploiement ${ }^{8}$ ». Ces fictions contemporaines se caractérisent selon lui par leur symbolisme soft : elles visent à " compenser la platitude de la réalité empirique ${ }^{9}$ " et à réinjecter du sens dans le matérialisme actuel sans verser pour autant dans les débordements de l'irrationnel.

En faisant des années 1980 le point de départ d'une contemporanéité détachée des grands récits de la modernité, la critique littéraire situe les littératures québécoise et française contemporaines sensiblement au même point tournant, avec ce resserrement sur l'intime entre autres caractéristiques. Le quotidien participerait du mouvement: s'y intéresser revient, semble-t-il, à tourner le dos aux "grands récits " idéologiques et romanesques et, du même coup, à épouser le «mode mineur» des fictions intimistes. Il se voit ainsi confiné par la critique à la sphère privée, domestique, sphère à la fois subjective et concrète, empreinte de «modestie», de «discrétion» et de «délicatesse». Un tel arrimage ne va pourtant pas de soi. D'une part la valeur que l'on accorde au quotidien est parfaitement réversible (dans le sens où l'on peut aussi bien considérer que raconter le quotidien est un rétrécissement de l'univers romanesque), d'autre part l'intériorité mi-subjective, mi-matérielle qu'on lui prête trouve, dans les incidents publics et collectifs auxquels s'intéresse Annie Ernaux dans ses chroniques, un contre-exemple suffisant pour ébranler l'évidence

$$
++
$$

4 Lori Saint-Martin, «Le métaféminisme et la nouvelle prose féminine au Québec», Voix et Images, vol. XVIII, no 1, automne 1992, p. 78-88; «Le corps et la fiction à réinventer : métamorphoses de la maternité dans l'écriture des femmes au Québec», Recherches féministes, vol. VII, n² 2, 1994, p. 115-134. 5 Pierre Nepveu, "Vers une nouvelle subjectivité», Québec Studies, vol. XX, printemps-été 1995, p. 42-46. 6 Pierre Nepveu, art. cité, p. 44. 7 Andrea Oberhuber, «La maison étrangère d'Élise Turcotte», Gilles Dupuis et Klaus-Dieter Ertler (dir.), À la carte. Le roman québécois (2000-2005), Frankfurt, Peter Lang, 2007, p. 429. La citation est tirée de Pierre Nepveu, «Les choses vivantes d'Élise Turcotte», Lectures des lieux, Montréal, Boréal, coll. «Papiers collés», 2004, p. 216. 8 Michel Biron, «Le symbolisme soft», Voix et Images, vol. XXVIII, nº 2, hiver 2003, p. 167. 9 Michel Biron, art. cité, p. 168. 
$d^{\prime}$ une telle circulation intime ${ }^{10}$. Le quotidien est non pas une sphère distincte de la réalité mais une construction imaginaire et narrative qui demande à être analysée. L'examen s'impose d'autant plus que, dans le cas du roman d'Élise Turcotte, le quotidien recouvre aussi bien l'ancrage dans la réalité concrète que la figuration symbolique du monde matériel. Comme le précise le prière d'insérer, «cette délicate attention au monde [...] compose un hymne à la grandeur retrouvée du quotidien». Qu'est-ce qui se joue dans cette grandeur retrouvée? Faut-il considérer le quotidien comme la valorisation de la vie matérielle ou comme le dépassement d'une réalité empirique somme toute triviale? Je m'intéresserai ici à la construction narrative du quotidien entre fragmentation temporelle et durée, entre images, motifs et événements. Je me demanderai également si la construction du quotidien en mode mineur ne relève pas d'un geste d'intériorisation qui, au-delà de la subjectivité à laquelle il se rattache, s'avère plus romanesque et merveilleux qu'on a pu le prétendre.

\section{FRAGMENTATION}

\section{DISSOCIATION DES INSTANTS}

L'ancrage dans le quotidien est, pour commencer, affaire de temps. Le découpage du roman met de l'avant un présent actuel. Les quatorze chapitres brefs de chacune des cinq parties morcellent le temps en autant d'aujourd'hui et de maintenant dont la récurrence ressortit à une quotidienneté élémentaire, quasi immédiate, insistant toutefois non pas sur ce qui revient, mais sur la disjonction des présents coupés les uns des autres. Les instants isolés font du quotidien une somme de jours autour desquels se distribuent hier et demain, avant et après. Tout comme l'univers gravite autour de la maison d'Albanie et Maria («ici»), le temps tourne autour d'un présent toujours recommencé. Le quotidien tient de l'attraction universelle qu'Élise Turcotte thématise dans son roman, il est le noyau de l'univers ici, aujourd'hui, maintenant ${ }^{11}$. On observe toutefois, dans la découpe du temps, une tension entre fragmentation et convergence des instants qui, dans les deux premières parties, penche nettement du côté de la première.

La répartition du temps en présents juxtaposés les uns aux autres s'effectue dans une écriture aux antipodes de la fluidité. Les chapitres brefs (d'environ deux à trois pages) composant chacune des cinq parties sont à leur tour divisés en blocs de paragraphes espacés par un blanc. Ces divisions internes permettent, entre autres, de dissocier les moments d'une même journée en autant de «maintenant», comme

$$
+++
$$

10 Annie Ernaux, Journal du dehors, Paris, Gallimard, coll. «Folio», 2000 [1993], 106 p. ; La vie extérieure, 1993-1999, Paris, Gallimard, coll. «Folio», 2001 [2000], 146 p. 11 Le motif de la planète et des choses qui tournent, qui circulent autour, revient à maintes reprises dans le roman et, comme la critique l'a signalé, les sauts entre les petites choses et le tout de la vie, entre l'ici-maintenant et l'univers, sont fréquents. Albanie souligne d'ailleurs explicitement cela : «Je pensais qu'une chemise mal attachée était liée au désordre de l'univers. » (BCV, 71) Notons au passage qu'une telle phrase est caractéristique du minimalisme de Turcotte, chez qui les menus détails du quotidien et le grand ordre (ou désordre) universel sont symboliquement liés (il faut en effet que la chemise mal attachée symbolise le désordre pour qu'elle se «rattache » à l'universel). 
dans le chapitre deux de la deuxième partie: «Maintenant, nous pouvons passer cette journée ensemble, à faire semblant d'être malade./Nous avons appris tout à l'heure qu'il y avait eu un autre tremblement de terre, cette fois en Algérie.» ( $B C V$, $60)$; «Maria veut maintenant que je dessine une mappemonde.» $(B C V, 63)$

La dissociation des instants s'accentue du fait qu'ils sont reliés à une activité concrète. Ce sont les occupations et les gens qui ordonnent le temps, faisant de l'ici et du maintenant un territoire habité et pratiqué. Le chapitre trois de la première partie est, à cet égard, significatif: le temps de l'horloge se divise en occupations distinctes, mais l'emploi du temps de la mère se décline en fonction de celui de sa fille, la journée se répartissant entre sa tâche de bibliothécaire et les activités de Maria gardée par sa grand-mère:

Aujourd'hui, à la bibliothèque, je dois ranger par ordre alphabétique toute une série de livres de recettes [...]. Dix heures: Maria écoute Les Contes de la forêt verte. Deux heures: elle fait du bricolage [...]. Trois heures: je range maintenant la section $\mathrm{B}$ des romans. (BCV, 18-19)

L'heure du souper: un cirque, comme d'habitude. Mais l'heure du bain se déroule comme un charme à cause des poissons qui accompagnent Maria. $(B C V, 19)$

Tout en montrant à quel point la mère est littéralement occupée par sa fille, ce dispositif ramène la journée à une juxtaposition d'instants (d'activités) disjoints. On saute de dix à deux heures et si, au bout du compte, on peut se faire l'idée d'une journée ordinaire d'Albanie, c'est que le découpage repose sur un scénario commun que les exemples concrétisent, entre habitudes et singularités.

\section{IMAGES DU TEMPS, EMBLEMES DE LA PERTE}

Si les choses de l'existence ordonnent le temps, elles le projettent aussi dans un présent atemporel, celui des images, des «il y a», des "c'est», des «toujours» et «jamais». Les choses alors s'empilent comme autant de petites vérités tournées vers l'essentiel, vers «ce qui compte vraiment»: «On peut vouloir tout raconter par le détail. Ou tout résumer. Ne garder que ce qui compte vraiment. Les odeurs. Le papier. L’homme qui est resté quatre jours sous le pont effondré. Sa survie. Celle des six cent personnes sans maison. Ce qui nous ramène encore aux enfants, seuls dans les ruines, en Arménie.» $(B C V$, 57) L'énumération accole ici les sensations minuscules (odeurs, papier) à une actualité catastrophique qui, en l'occurrence, revient, introduisant le temps piétinant de la fixation obsessionnelle. Dans l'image, et plus encore dans l'image qui revient, les catastrophes se figent, elles sortent de l'actualité pour devenir l'emblème du désordre du monde et de ses victimes innocentes. En intériorisant les images télévisuelles qui «s'empilent dans [son] cerveau» $(B C V, 12)$, Albanie introduit l'Autre dans sa tête non sans faire de celui-ci, et de toutes les catastrophes, un symbole du penchant mortifère qui la ronge et ruine l'univers. Ces images "sont un exemple de ce qui est malade dans la vie» $(B C V, 80)$, elles figurent 
la séparation. La thématisation de ce qui s'écroule contribue d'ailleurs, dans les deux premières parties surtout (où les images sont envahissantes), à renforcer l'effet de disjonction tout en lui donnant un sens éminemment symbolique: "Tout se défait, les choses ne tiennent plus ensemble» $(B C V, 41)$, dit Albanie à propos des «jours de garderie», qui sont l'image de la séparation à l'échelle quotidienne. Chaque matin de déchirement à la garderie renvoie au tout des choses, offre une image de la perte dans laquelle Maria, la Terre et les enfants victimes s'amoncellent:

\begin{abstract}
Autre image de la perte, plus tard, avec l'enfant dans mes bras. Du haut d'un escalier, faux pas, je dégringole, l'enfant si petit tombe, la Terre cesse de tourner. Ou encore : affiches avec les photos et les noms des enfants disparus : idées folles de ce qu'ils sont devenus, de comment ils sont morts; enlèvement; viols; maladie ; abandon. Ou encore : ça pourrait être Maria, un bébé étranglé, un bébé perdu, ça pourrait être Maria. $(B C V, 23)$
\end{abstract}

Dans cette image mentale, «l'enfant» Maria et les victimes, le drame privé imaginaire et les disparitions publiques sont listés sur le mode de la juxtaposition, que l'on peut dégager comme un des traits de l'écriture de Turcotte. Celle-ci relève d'un mode parataxique supprimant toute liaison entre le petit (microcosme) et le grand (macrocosme), toute articulation entre l'extérieur (les affiches) et l'intime (moi et Maria). D'emblée, nous avons affaire à quelque chose qui s'ajoute - l'«Autre image» de la perte succède à une "première image de la perte» $(B C V, 22)$; la phrase nominale permet au lecteur de rapidement visualiser l'image sans passer par les détours de la syntaxe. Cette image vaut comme exemple et les exemples, assez nombreux dans Le bruit des choses vivantes, se juxtaposent eux aussi pour faire du monde une collection emblématique signifiante.

Autant les images télévisuelles envahissent la conscience d'Albanie, autant cette dernière projette son univers intérieur (imagination, rêves, visions) tantôt comme un petit film, tantôt comme les photographies d'un album ${ }^{12}$. Toutes ces images isolent un moment, un geste, un objet quotidien pour le donner en exemple, en faire le symbole d'une réalité abstraite (désir, peur, perte, etc.): le meurtre d'un enfant, c'est le «meurtre du monde», les chaussures, c'est le désir d'Albanie et l'idée du désir, etc. Les images arrachent les choses au temps tandis que le temps se figure dans une image : Albanie le compare à «une voiture qui passe et qui ne laisse pas de chance» $(B C V, 92)$. Ce petit film mental n'est pas si loin de la séquence de dégringolade dans les escaliers; il met en scène, encore une fois, la disparition ${ }^{13}$. Ce type

$$
+++
$$

12 «Il faudrait que notre tête soit un immense album photographique.» $(B C V, 54)$ Corinne Larochelle poursuit dans cette veine: "De par sa narration fragmentée, fractionnée en "morceaux de vie" ( $B C V$, quatrième de couverture), Le bruit des choses vivantes se lit comme on feuillette un album photo.» ("Lire l'image: Le bruit des choses vivantes d'Élise Turcotte», Voix et Images, vol. XXIII, n 3, printemps 1998, p. 553.) Ajoutons que les représentations qu'Albanie se fait d'elle-même et de ses proches sont également filmiques («Je suis peut-être en train de devenir la personne cachée qui les [Félix et Agnès] regarde vivre. Je vois leurs gestes dans un film muet.» $[B C V, 79]$ ) et littéraires ("Quand Maria n'est pas là, les heures se déroulent dans un seul souffle, elles ne sont pas divisées en chapitres comme une histoire.» $[B C V, 45])$. 13 La figuration concerne, en l'occurrence, Maria qui «commence à comprendre ce qu'est le temps» $(B C V, 92)$, mais c'est aussi l'anxiété de sa mère qu'elle révèle. 
de figuration par métaphore ou comparaison abonde dans le roman: " voilà comment la peur peut faire un trou dans le présent» ( $B C V, 53$; je souligne); "Je ne sais pas comment être cette joie derrière le mur, ni cette peine [...].» (BCV, 72 ; je souligne) Là où les images des petites choses renvoient à de grandes vérités abstraites, les abstractions sont figurées par les petites choses de la vie, le plus souvent spatialisées. Tout bruisse, tout murmure, tout signifie dans un processus imageant incessant qui gravite autour de deux noyaux existentiels à valeur universelle: la mort et la vie.

La poétique de la juxtaposition participe de ce partage symbolique entre vie et mort. Si l'addition des images donne un poids certain à une telle division du monde, l'esthétique fragmentaire amène également le lecteur à expérimenter la disjonction des éléments du récit qui, en cela, penche significativement du côté de la mort. Images et exemples sont additionnés, agglutinés en groupes phrastiques que ponctuent des deux-points explicatifs et des points-virgules énumératifs caractéristiques de la parataxe. Cette poétique tient à cette manière de découper le monde en sujets ${ }^{14}$ et en instants disjoints, manière qui trouve dans la ponctuation, les blancs, les divisions, les phrases nominales, les exemples, les énumérations et la liste $(B C V, 38-39)$, un style significatif. Toutefois - et puisque la mort a pour pendant la vie - , la fragmentation du récit est aussi ce qui permet de relier les choses, de créer des ponts entre elles.

\section{LIENS}

\section{DURER}

Revenons au temps. Le cumul des instants et la progression à coups de "plus tard» insituables dissocient des moments quasi photographiques. Les images intériorisées du présent se donnent cependant - et de plus en plus à mesure que l'on progresse dans le roman - par rapport à un avant ou un après de sorte à ébaucher une durée, celle que Maria commence à apprendre, et sa mère, à réapprendre:

j'ai encore rêvé à elle: on me l'enlevait. $(B C V, 53)$

Le rêve où je visite une maison est revenu plusieurs fois au cours des dernières semaines. $(B C V, 107)$

$$
+++
$$

14 Cela comprend les personnages, qui surgissent «dans la tête» d'Albanie comme autant d'images séparées: «Jeanne./Elle veut surtout savoir ce qui s'est passé avec Alexandre. » $(B C V, 30)$; «Le père de Maria./On pouvait dire avant: Albanie est bien, Albanie est heureuse.» $(B C V, 43)$; «Alexandre et moi./Les mots viennent d'ailleurs.» $(B C V, 45)$ Ces phrases nominales isolant un sujet en allant à la ligne fonctionnent sur le mode de la légende photographique: elles désignent le «qui» tout comme, par ailleurs, elles étiquettent le «quand» («Plusieurs jours de congé./Nous en profitons pour ranger les vêtements trop petits de Maria.» $[B C V, 90])$, le "où » ( $\mathrm{Au}$ bar./Les choses murmurent autour de moi [...].» [BCV, 25]), le "quoi» (« Je fais un rêve./Nous visitons une maison qui ressemble à celle de notre cahier. » $[B C V, 96])$. Bien distincts de la mise en retrait qui charge certains élément d'accents affectifs (accents qu'Élise Turcotte n'hésite pas à multiplier), ces retours à la ligne cadrent et découpent la réalité comme des choses séparées. 
Nous n'avons plus de crème aux agrumes. Nous décidons d'attendre le printemps pour en acheter une autre bouteille. $(B C V, 109)$

Depuis qu'elle sait que je l'ai vue de ma fenêtre, Maria n'a plus tellement peur à la garderie.» $(B C V, 123)$

Les mots continuent à grandir autour de nous. [...] La vie est plus lente. $(B C V, 173)$

Alors que le retour des rêves marque un temps étale qui piétine, une durée s'installe à partir des choses de la vie. Dans les deux derniers exemples, elle apparaît de manière positive: on grandit, de même que les mots autour. La séparation à la garderie est désormais possible sans la peur (celle de Maria faisant écho à celle d'Albanie), sans que le lien ne se rompe. La durée se mesure à des acquisitions concrètes, petites conquêtes au quotidien sur la perte et «le meurtre du monde». L'enfant symbolise ce temps qui dure et grandit, elle incarne l'apprentissage de la vie.

En intériorisant le point de vue de l'enfant dont elle est aussi le témoin, la mère renoue avec le récit d'initiation. Le quotidien devient une série de commencements ${ }^{15}$. Les premières fois marquent les instants d'une pierre angulaire qui, au contraire du vide, «[bâtit] la mémoire» $(B C V, 69)$ :

Ce sera le premier Noël double de Maria. $(B C V, 69)$

Aujourd'hui, j'ai senti pour la première fois un vent chaud et léger en ouvrant la porte. $(B C V, 152)$

Notre premier sujet de conversation est donc facile à trouver : l'absence de Maria, son père et tout ce qui s'ensuit. $(B C V, 176)$

Ensuite, je suis allée dans le parc devant la bibliothèque pour dîner et c'est là qu'Agnès est venue me rejoindre pour la première fois. $(B C V, 210-211)$

Le temps cyclique des saisons, des fêtes et des jours se trouve relancé dans une série d'initiations amorçant autant d'histoires. Il faut prendre au sérieux l'équivalence qu'Albanie fait entre les premières fois et les histoires à propos des habits trop petits de Maria qu'elle range dans des boîtes : «À la fin, chaque vêtement possède une histoire. Chaque robe, chaque pyjama est lié à une première fois. » $(B C V, 91)$ Les choses se lient une fois racontées. Les histoires conjurent l'insignifiance du quotidien, elles introduisent la moindre chose dans le temps des commencements orientés vers une suite, une transmission ${ }^{16}$. Contrairement à la boîte à chaussures ne contenant qu'une

$$
++
$$

15 Turcotte s'écarte par là du lieu commun du quotidien comme routine, habitude, répétition. 16 AnneMarie Clément signale la segmentation ostensible du roman d’Élise Turcotte qui, par là, met en avant une fragmentation et une discontinuité caractéristiques du contemporain. Mais la juxtaposition et l'addition des petites histoires du quotidien, avec la logique associative qui s'y rattache, ne débouchent pas sur un éclatement du sens. 
désastreuse disparition, les boîtes à vêtements sont remplies des "fragments brillants du temps que nous avons eu jusqu'ici» $(B C V, 91)$. Ranger, mettre de l'ordre dans le chaos de l'univers, c'est alors mettre le temps en boîte sous forme de récits qui opposent à ce qui se dérobe autant de jalons mémoriels - augures d'avenir. Les vêtements trop petits symbolisent et le passage du temps et sa mise en ordre dans des histoires: ils sont désormais précieux, aussi «brillants» qu'étoiles, diamants, trésors... Dans ce contexte, les saisons ne reviennent pas vraiment. À l'intérieur de la durée d'une année qui délimite le roman, elles sont surtout autant de premières fois allant grosso modo du froid vers le chaud. Les feuilles d'automne jonchant le sol dans la première partie figurent la perte, tandis que l'éclosion des fleurs, dans la quatrième, marque le retour à la vie. Ce symbolisme galvaudé a ceci d'intéressant qu'il ne ramène pas le «tremblement de bonheur» $(B C V, 245)$, sur lequel se ferme le roman, à un aboutissement définitif: après l'été revient l'automne, après le jour, la nuit, etc. Se trace néanmoins, en pointillé, un parcours linéaire qui, de l'automne à l'été, de l'angoisse de la perte au départ d'Albanie et de Maria dans le Nord rêvé, fait du roman tout entier une initiation au voyage, une première fois déterminante saisie dans l'instant du départ, dans le tremblement de ce qui vient ${ }^{17}$. De l'évanouissement des souliers emblématiques du désir à la réalisation du désir, quelque chose progresse et s'accomplit. Le sens d'une histoire en marche passe par l'intégration du voyage comme une promesse jurée à Maria. Sans en faire son unique préoccupation, Albanie y revient de loin en loin: le voyage oriente le récit, en devient un des fils conducteurs. Comme tout objectif, le «rêve de voyage » $(B C V, 65)$ contribue à unifier le roman. Pourtant, parce que le voyage n'est pas la seule histoire en cours, la trame narrative générée par la progressive «[entrée] dans [le] rêve» (BCV, 200) se dissémine et se démultiplie d'autant.

\section{GRANDIR}

L'élargissement progressif du cercle intime contribue cependant lui aussi à lier le récit. Alors qu'au début seuls Jeanne et son fils Gabriel font partie du territoire d'Albanie, celui-ci s'ouvre peu à peu aux amants - Alexandre puis Pierre - , au petit voisin Félix et à son père, à Agnès, l'habituée de la bibliothèque. Par là, l'univers d'Albanie et de Maria grandit: il se déploie dans la durée, s'étend tout en multipliant les histoires secondaires. Celles-ci prennent le dessus sur les images spectaculaires et déconnectées du début, elles tracent un espace de proximité plus actif et investi. Plus on avance dans le roman, plus Albanie fabrique des images

$$
++
$$

Elles permettent plutôt, remarque fort justement Anne-Marie Clément, «une compréhension narrative qui se sait imparfaite, [...] une constitution de la mémoire (et donc d'une temporalité) qui permet — malgré tout, c'està-dire malgré la fragmentation du temps - de se situer dans le temps, et [une] transmission qui s'accomplit par la communication des récits». (A.-M. Clément souligne.) Voir René Audet et Andrée Mercier (dir.), "La narrativité à l'épreuve de la discontinuité», La narrativité contemporaine au Québec, vol. 1, La littérature et ses enjeux narratifs, Québec, Presses de l'Université Laval, 2004, p. 121. 17 D'autant plus que le tremblement de bonheur répond, en écho, au tremblement de terre en Algérie et à celui qui est souvent relié à la peur dans le roman. 
(dessinées, photographiques, vidéo, mentales). La première rencontre avec Pierre est un échange muet dans lequel elle produit l'image qu'elle veut graver dans la tête de cet homme encore inconnu:

Ce n'est pas tout. Je sens qu'il y a autre chose, que le hasard se tait pour l'instant, mais qu'il connaît déjà cet homme qui vient de franchir le seuil de la porte, en face de chez nous, chez Félix. [...]

L'homme nous voit, regarde sur une feuille qu'il a sortie de sa poche, nous regarde encore en plissant les yeux puis s'en va. [...]

C'est pour ça que mon geste à moi a été de prendre Maria dans mes bras pour lui montrer que je suis forte et aimante. J'ai pris Maria dans mes bras pour que cette image reste bien gravée en lui. Pour que cela fasse un contraste avec ce qu'il a trouvé chez Félix. Pour que la vie soit possible sur cette planète et pour qu'il revienne. $(B C V, 162)$

Chargée de suspense, cette première fois tend vers une suite qui se nouera peu à peu dans une histoire. Parce que cette histoire-là, comme celle de Jeanne et de Jacques, raconte la possibilité de l'amour après la séparation - c'est-à-dire, pour reprendre les termes d'Albanie, la possibilité de la vie -, l'image gravée dans la tête de l'inconnu qui va revenir apparaît bel et bien comme ce qui fait lien. Sa reprise ultérieure précise la possibilité d'un commencement: «Cette fois, je ne sais pas si cet homme aperçu de l'autre côté de la rue sera un homme dans ma vie, mais je suis sûre qu'avec cette image de moi et ma petite fille dans les bras, il n'y aura pas de faux départs. » $(B C V, 165)$ La situation évolue et l'éventualité d'un vrai départ génère une tension narrative de l'ordre du suspense. L'alternance des histoires secondaires alimente ce suspense sur le mode du report, elle distribue la durée dans le kaléidoscope agrandi et démultiplié de la maison d'Albanie et de Maria, ce noyau autour duquel gravitent de plus en plus de «mondes».

\section{TRESSER}

La signification des choses tient autant à leur implication narrative dans une durée qu'aux réflexions d'Albanie: «J'ai toujours pensé que les choses étaient liées, même si c'est parfois par un fil invisible [...].» $(B C V, 71)$; "C'est toujours le même problème, les liens, les liens que nous ne pouvons pas faire, et que nous devons faire entre les choses, entre l'assassinat lent, profond, et la vie qui est une promesse faite à ma petite Maria.» $(B C V, 135)$ On retrouve, dans cette thématique des liens, la lutte entre mort et vie à laquelle renvoie symboliquement l'entièreté du roman. La reprise réflexive des mêmes thèmes contribue à orienter le sens du récit, à le faire converger autour des grandes questions obsédant Albanie.

À cette thématisation qui participe du geste d'intériorisation correspond un travail du motif, travail de détail qui tresse des liens non narratifs. Le motif du fil 
invisible, par exemple, se retrouve dans le dernier chapitre, avant l'ultime tremblement de bonheur : «Et peut-être qu'un jour nous serons tous sur cette plage, le cœur un peu plus léger et un peu plus lourd à la fois, tous reliés par un fil invisible. » ( $B C V$, 245) Ce fil tisse une cohésion discontinue, verticale ${ }^{18}$. D'une occurrence à l'autre, son sens se déplace, se déploie: il relie finalement non plus «les choses» mais les personnes, et son invisibilité n'est plus, comme à la page 71, un moindre mal. C'est une qualité en soi qui, dès lors, renvoie de manière analogique au schème de ce qui est caché, présence secrète qui anime les choses et les êtres, comme dans cette image qu'Albanie découvre: "Je m'assois près d'elle et je découvre aussitôt cette scène à l'intérieur de moi: Agnès lisant une lettre, assise sur les marches de la bibliothèque, une larme coule sur sa joue, mais ce n'est pas comme si elle pleurait [...].» $(B C V$, 153) Les visions d'Albanie révèlent une essence, elles isolent des instants pour en faire le symbole de ce qui, au fond, résume Agnès ou Félix (pour elle, une larme réconciliée avec le monde, pour lui, la solitude et l'abandon): "Pour Félix, nous savons déjà ce qu'il y aura toujours à l'intérieur de lui: un petit enfant qui trottine derrière son père. L'homme ne se retourne pas. Seul, le petit garçon continue à pleurer.» $(B C V, 151)$ Les visions de ce qu'il y a à l'intérieur semblent tout droit sorties de scènes quotidiennes, mais parce qu'elles figurent une essence invisible, elles arrachent les gestes à toute contingence pour en faire le lieu d'une révélation.

La reprise des motifs renvoie aussi à une même source énonciative autour de laquelle se rassemble le discours morcelé. Aux détails relativement accessoires, tel le «fil invisible», correspondent des motifs centralisateurs qui convergent vers de grands thèmes. Ainsi en va-t-il des souliers évoqués plus haut:

Images de la peur d'Albanie.

Au commencement, il y a peut-être l'histoire des souliers dérobés. Première image de la perte: j'avais cinq ou six ans, je ne sais plus. Les souliers étaient en cuir verni noir, les plus beaux que j'avais jamais vus. Pour moi, premiers souliers de grande fille. En plus, ils brillaient. Je ne les ai jamais portés.

Nous les avions rangés dans la garde-robe de l'entrée, maman et moi, ils étaient là, bien en vue, ils brillaient même dans le noir. Mais le lendemain, plus de souliers. Et surtout: personne n'a jamais compris ce qui avait pu se produire.

Les souliers sont maintenant devenus l'emblème du désir. Et dans le désir, il y a la peur qui est l'emblème de la disparition. (BCV, 22-23)

Dans cette autre histoire de commencement, l'image des souliers représente le désir à travers les yeux de l'enfant; elle en devient l'emblème et, parce que les souliers

$$
++
$$

18 L'idée d'une verticalité du motif (par opposition à l'horizontalité narrative) correspond à l'image qu'Élise Turcotte donne de son écriture: «Les plans, je les fais à mesure. En fait, ce ne sont pas des plans, du moins pas des plans linéaires; ce sont des plans verticaux. J'écris à l'ordinateur, mais j'ai de nombreux cahiers de notes, et j'établis des correspondances, des liens entre tel symbole, tel personnage, etc. [...] Aussi ai-je un peu l'impression de faire une "tapisserie" [...] avec sa texture, son architecture particulière, où tous les motifs sont enchevêtrés. » (Denise Brassard, «Entretien avec Élise Turcotte», Voix et Images, vol. XXXI, n 3, printemps 2006 , p. 22. 
ont disparu, elle permet d'associer ce désir à la peur de la perte. Le motif des souliers est d'emblée réfléchi, étiqueté, thématisé comme un emblème pour ainsi dire à double fond. L'enfant Maria réveille en Albanie l'enfant qu'elle fut, elle ranime l'esprit enfantin faisant des souliers une merveille. S'y greffe le motif de ce qui brille "même dans le noir». Quand, à propos de ce qui ne marche pas avec Alexandre, Albanie remarque que « [l]e matin, [elle] [se] lève, espérant trouver un mot d'amour dans [ses] souliers. Il n'y en a jamais» $(B C V, 29)$, il est clair que quelque chose manque. Ensuite, quand elle découvre que Maria «a placé toutes ses billes dans un de [ses] souliers", elle compare celles-ci avec "des planètes sur fond noir» ( $B C V$, 72): le lien avec les souliers vernis s'impose comme un clin d'œil qui «n'arrête pas de $[\ldots .$.$] faire signe» (B C V, 72)$, il ouvre sur le cosmos par le biais des planètes, autre motif. Beaucoup plus loin, alors qu'elle vient d'apprendre que l'inconnu rencontré devant la maison de Félix s'appelle Pierre, Albanie a, en le quittant, une vision dont la portée symbolique est évidente: «Je me retourne pour prendre la direction opposée, et alors l'image des billes qui me regardent dans le fond noir d'une chaussure me revient./Je prends Maria par la taille, je dis, je sais ce qu'on va faire, on va s'acheter des souliers pour le printemps.» $(B C V, 166)$ Dans l'économie du récit, l'achat de nouvelles chaussures figure un nouveau départ, un recommencement printanier, à la suite et à la place des souliers dérobés. Enfin, lorsque, le jour de sa fête, Maria dit: «maman les billes sont encore dans ton soulier» $(B C V, 201)$, le motif s'installe dans une durée qui, contrairement à ce qui s'est d'abord dérobé, reste en place. Cela coïncide avec la surprise qu'Albanie réserve à Maria - les billets pour entrer dans leur «rêve de voyage» - et croise le symbole de la place vacante qu'il faut toujours laisser dans une valise: "cette place, c'est l'endroit où se trouvera le trésor, un coquillage ou n'importe quel autre souvenir; c'est l'endroit où se trouve le désir " $(B C V, 225)$. Ce symbole renverse complètement l'emblème des souliers dérobés: là où, "au commencement», la disparition involontaire tuait le désir, la place vacante est une promesse tournée vers l'avenir; au cœur de ce désir loge non plus la perte mais la possibilité d'un trésor de mémoire. À partir de ce seul motif, on voit bien en quoi le départ d'Albanie et Maria dans le Grand Nord est vraiment un départ: il va dans le sens d'une vie de chaque instant, où les souvenirs font lien.

Les motifs tissent donc une cohésion textuelle signifiante qui finit par rejoindre la trame d'un récit. À travers eux, les histoires secondaires se rassemblent autour du noyau mère-fille. Le minimalisme narratif tient déjà à ce tressage de motifs minuscules qui, par un minutieux travail de reprise, finissent ici par s'impliquer dans la durée. Le roman rassemble plusieurs lignes de sens à partir de petites choses: billes dans un soulier, valises, fleurs. Comme dans les romans minimalistes de Jean-Philippe Toussaint, Élise Turcotte fait des motifs minuscules le moteur d'un récit intériorisé, mais contrairement à lui, elle ne le pousse pas du côté de l'épopée du dérisoire ${ }^{19}$.

$$
++
$$

19 Warren Motte qualifie l'œuvre minimaliste de Jean-Philippe Toussaint en ces termes: "La Télévision takes its place in a droll and highly original body of work that is beginning to look like an epic of the trivial.» («TV Guide», Neophilologus, n 83, 1999, p. 529.) ("La télévision s'inscrit dans une œuvre drôlatique très originale qui commence à ressembler à une épopée de l'insignifiant.» [ma traduction]) 
Turcotte fait plutôt du quotidien le départ d'une vectorisation narrative signifiante, où la moindre des choses acquiert un sens et une valeur symboliques. Par là, elle est plus proche du minimalisme de Philippe Delerm que des distorsions ludiques de Jean-Philippe Toussaint. À l'instar de Delerm, elle associe les images à l'intériorisation d'un sens, à la fixation d'une trace, et elle célèbre l'extraordinaire de la vie de tous les jours. La valorisation de l'espace intime et le rejet du caractère insaisissable de la réalité se retrouvent dans les deux cas. Turcotte ne passe cependant pas par une esthétisation du monde comparable à celle de Delerm: ses références culturelles renvoient à la culture américaine populaire, et l'esthétique intervient surtout à travers les dessins de Maria, autrement dit à travers l'enfance de l'art. L'expérience vécue est au cœur des «petits miracles» de chaque jour, alors que pour Delerm, l'impression se fonde dans un savoir partagé coupé de toute subjectivité assumée, de toute transformation du rapport au monde. Cette transformation passe, chez Turcotte, par une prise de conscience de la perte et l'apprentissage d'un «comment vivre", tandis que la perte reste, chez Delerm, une mélancolie sous-jacente aux petits bonheurs fugitifs. Les microcosmes d'Enregistrements pirates ${ }^{20}$ sont des univers immobiles, suspendus hors de la durée, tandis que Le bruit des choses vivantes engage la durée et le passage du temps.

\section{INTRIGUE}

\section{SuRgisSEMENT DE L'ÉVÉNEMENT}

Si ce roman ancré dans le quotidien s'écarte des aventures et des péripéties caractéristiques du genre, il se noue dans une intrigue qui, aussi ténue soit-elle, engage une trame et un imaginaire bel et bien romanesques.. L'intrigue n'est pas première. Cependant, à partir du moment où, autour du drame de Félix, différentes lignes de récit émergent et se recoupent, la cohésion narrative prend une tournure plus conséquente. Dans le chapitre neuf de la troisième partie (ÊTRE TÉMOIN), un événement déterminant a lieu:

À ce moment-là, nous avons été témoins d'une chose qu'il ne sera plus jamais possible d'ignorer.

Nous avons vu Félix, un petit garçon de trois ans, entrer tout seul dans la garderie. Il a fait le chemin tout seul, il a attendu que quelqu'un passe derrière lui pour ouvrir la porte, il a tourné à gauche dans le corridor de la vieille école, puis il a trouvé sa classe et il est entré.

Il a vu la feuille collée sur sa case mais il n’a rien dit.

$$
++
$$

20 Philippe Delerm, Enregistrements pirates, Monaco, Éditions du Rocher, 2003, 147 p. Pour une analyse de la mise en récit du quotidien dans ce recueil, voir mon article «L'ordinaire et le bref: les instantanés de Philippe Delerm", Catherine Douzou et Lise Gauvin (dir.), Frontières de la nouvelle de langue française. Europe et Amérique du Nord (1945-2005), Dijon, Éditions universitaires de Dijon, coll. «Écritures», 2006, p. 13-24. 
L'événement s'est produit, et comme tous les événements, il va continuer à se produire ailleurs et sans nous. Mais c'est à partir de lui que nous allons commencer à savoir des choses sur Félix.

Lise a dit, maintenant c'est à moi d'agir. (BCV, 132-133)

Le contraste entre la dramatisation de l'événement et son apparente banalité repose implicitement sur le scandale qu'il y a, pour un enfant de trois ans, à faire tout seul le chemin de la garderie. Il s'agit d'un scandale commun, ordinaire, aux antipodes des images spectaculaires de viols, d'enlèvements qui hantent Albanie mais qui, tout comme elles, représente l'abandon. L'événement du petit Félix laissé à lui-même se détache très vite de la contingence spatio-temporelle, il est exemplifié idéalement, capable qu'il est de s'actualiser «ailleurs et sans nous». Entre Félix et «la souffrance des enfants» $(B C V, 23)$, le lien s'effectue d'emblée : le petit garçon incarne la victime innocente qui obnubile Albanie et la rend insidieusement coupable: "Cet été, un petit garçon s'est noyé dans la piscine. Publique. L'adulte avait dit au garçon de quatre ans de surveiller son frère de dix-huit mois. Il tombe dans la piscine. Personne ne le voit. [...] Corps mou, si petit. On le retrouve. On crie. On sait que tout est de notre faute.» $(B C V, 39)$ L'intertexte chrétien semble investir ici la figure de l'innocent sacrifié et le sentiment de la faute, mais avec le petit Félix, parce que l'événement arrive ici et pas ailleurs, il devient possible de se racheter. L'événement est aussi celui-là: alors qu'Albanie ne pouvait rien faire contre la souffrance des victimes innocentes, la vision du petit Félix déclenche l'action de Lise et la sienne. De témoin passif elle devient témoin actif, renouant alors, à partir du milieu du roman, avec la centralité de l'action. Certes, l'action se déploie à petite échelle, celle du drame local, mais elle renvoie symboliquement aux abandons majeurs et à la figure de la victime innocente (l'enfant). Pour le dire en termes minimalistes : less is more...

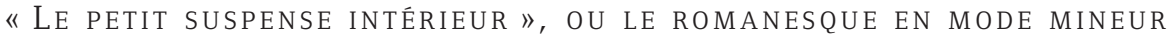

L'événement déclenche deux conséquences principales. D'abord commence l'enquête (écho à l'enquête imaginaire d'Albanie et de Maria ${ }^{21}$ ) qui va amener dans le voisinage un travailleur social chargé de trouver pour Félix une famille d'accueil. Ce nouveau venu, dont on apprendra bientôt qu'il se nomme Pierre, va réintroduire le désir dans la maison d'Albanie et lui donner l'impression de former une famille, où l'amitié et l'amour, les adultes et les enfants, se retrouvent réunis autour d'une grande tablée: «nous ressemblons à une sorte de famille dans une séquence de film américain. Des amis discutent, ils parlent de choses gaies, mais aucun ne parvient à faire taire son petit suspense intérieur.» $(B C V, 193)$ Le "petit suspense intérieur»

$$
++
$$

21 «Il [Félix] donne des coups de pieds sur la clôture. Ses souliers sont trop grands./Nous l'observons un peu, puis Maria me demande s'il attend sa maman./Peut-être que oui, Maria./Nous décidons d'aller lui demander./Il fait froid. Maria ne veut pas de mitaines, elle met ses mains dans ses poches pour faire grande. Elle dit, c'est comme une enquête de l'inspecteur Gadget.» $(B C V, 73)$ 
renvoie ponctuellement aux angoisses, aux inquiétudes, mais il définit bien la nature romanesque de l'intrigue minimaliste. De même que chaque convive garde en dedans les ressorts d'un drame, de même Albanie introduit un petit suspense intérieur en projetant la scène comme un film. Le caractère romanesque du repas relève d'une intériorisation, ce que redoublent les personnages eux-mêmes avec ce qu'ils gardent en réserve. La présence de Félix à la table n'est bien sûr pas fortuite. La honte ressentie par son père, lorsque, en revenant chercher son fils, il voit Pierre, les confidences de ce dernier sur la famille d'accueil qu'il a enfin trouvée pour Félix, l'espoir que cela représente pour le petit garçon, le désarroi de son père et, pour finir, son suicide par noyade, sont l'issue dramatique de l'événement déclencheur. Avec la mort du père de Félix annoncée dans les journaux, on rejoint les images médiatiques du début et le tragique de l'existence. L'histoire d'amour d'Albanie et de Pierre est l'autre conséquence de l'événement déclencheur. Romanesque en elle-même, cette histoire symbolise la vie où habiter ensemble et «grandir» est possible.

L'événement, aussi minuscule soit-il, débouche donc sur des rencontres et des drames qui n'auraient, autrement, pas eu lieu. Il place au centre du roman la figure de la victime et rejoue, dans sa trame, les couples abandon/amour, mort/vie. Le caractère romanesque de l'intrigue tient aux topoï impliqués (victime innocente, rencontre amoureuse, énigme liée à l'enquête) et, surtout, à la vision intérieure projetant la vie comme un film ou un roman chargé d'un "petit suspense». Car si l'intrigue aboutit directement à l'action, le romanesque, lui, découle de l'intériorisation du monde par Albanie. Les hasards et les coïncidences qui surviennent à la suite de l'enquête sont en effet tout intérieurs: "On ne saura jamais pourquoi les hasards nous bousculent, ni pourquoi une histoire tourne autour de nous et un jour atterrit à nos pieds $[\ldots]$.» $(B C V, 140)$ Le monde quotidien est lu comme un roman plein de surprises qui trace la ligne d'un futur. Après la rencontre avec Pierre, par hasard, «comme dans un film» $(B C V, 162)$, Albanie, persuadée qu'il va revenir, évoque l'accomplissement d'un destin: "une voix souffle les événements qu'on ignore» $(B C V, 163)$. Voilà qui ménage le suspense à partir $\mathrm{d}$ 'incidents ordinaires: «La vie est une coïncidence rare» $(B C V, 181)$, remarque Albanie. La transfiguration romanesque du quotidien est donc en elle-même une forme d'intériorisation. Elle se donne comme la vérité vraie qui dépasse la réalité superficielle, révèle le cœur des choses. Et ce cœur est aussi merveilleux.

\section{VISIONS ET MERVEILLES}

Le «rêve de voyage» qu'Albanie et Maria vont finalement réaliser marque l'absorption d'un merveilleux enfantin qui, à la manière du chiasme, renverse l'ordre établi ${ }^{22}$. À l'image de la dimension initiatique qui passe par la figure de l'enfant, le voyage intègre une perception enfantine. Le merveilleux, qui fait de la moindre petite chose un événement extraordinaire, "grandit» le quotidien à hauteur d'en-

$$
++
$$


fant, il le hausse à la (dé)mesure de ses joies et chagrins. Ce qu'Albanie intériorise, c'est aussi ce merveilleux semé dans le roman comme autant de billes reliant l'ordinaire et l'extraordinaire, le microcosme et l'infini, la réalité et le rêve (ou les histoires, ou les dessins, ou les images). Le rapport mère-fille implique à son tour l'intériorisation d'une catégorie générique - le merveilleux - qui enchante la maison et donne vie à toute chose. Albanie porte en elle ce regard d'enfant se devinant dans d'innombrables formules associant le quotidien et la merveille: "demain sera une journée normale [...] Nous mettrons nos tabliers, Maria et moi, et nous serons extraordinaires.» $(B C V, 37)$; «Nous vieillissons et chaque jour notre âme se révèle dans une petite phrase inouïe.» $(B C V, 138)$; «Maintenant, sur la vidéo, on peut voir la joie et la joie fait une ombre sur le mur. Elle se détache et devient une chose précieuse que l'on tient cachée dans notre paume, sous la mitaine, comme un billet d'autobus.» (BCV, 146-147) L'alliance du concret et de l'abstrait, du visible et de l'invisible, fait du quotidien le signe d'autre chose de plus vaste. Ainsi devient-il symbole, noyau, «chose précieuse » cachée à l'intérieur, sous la surface, tout au fond. Les boîtes s'ouvrent sur la joie ou le malheur sans basculer dans le fantastique ou le mysticisme. Le merveilleux, lui, ne déborde pas des frontières du vraisemblable: d'une part, il est intégré à une réflexion d'ordre métaphysique sur la vie, la mort, le temps et «l'être là» (que l'on peut attribuer à Albanie), d'autre part, il s'ancre dans l'expérience quotidienne d'une famille monoparentale où la vision enfantine insémine les méditations de la mère, y compris à propos des questions existentielles qu'elle se pose et de l'incessant désir de comprendre ce que «ça veut dire ${ }^{23}$ ». Albanie continue ainsi de porter sa fille en dedans tout en s'en faisant le témoin responsable (elle ne retombe pas en enfance). Le romanesque et le merveilleux dévoilent «le bruit des choses vivantes » $(B C V, 174)$ et, de l'intérieur, sans excès ni débordements, réenchantent le monde.

\section{PROFONDEUR ET LÉGËRTÉ DU QUOTIDIEN}

L'émergence du quotidien dans la littérature contemporaine engage une attention renouvelée aux choses, aux parcours et aux gestes de tous les jours dont la délimitation, le sens et la valeur varient considérablement d'un récit à l'autre. Chez Turcotte, le quotidien est un espace de transformation de l'expérience visant à redonner sens et centre à un monde fragmenté et tragique, à le réenchanter malgré tout. Cela passe par l'intériorisation d'un territoire à ordonner et à comprendre. Comprendre le monde, c'est accéder à une vérité essentielle, à «l'âme», au «noyau »,

$$
++
$$

23 Albanie ne cesse d'expliquer ce que "ça veut dire», qu'il s'agisse d'elle-même, de sa fille ou des autres: «J'ai dit, c'est le noyau de sa vie. Je voulais dire, le noyau de son être.» (BCV, 151); "Plus tard, juste avant de se coucher pour la nuit, elle emporte tous ses jouets dans sa chambre, les dépose au pied de son lit en me disant qu'elle n'est pas toujours certaine d'avoir quatre ans. Elle veut dire à toutes les minutes. Elle veut dire qu'il y a parfois une heure dans une journée où elle ne se sent pas bien dans son âge.» $(B C V, 206)$; «Puis Jacques nous a fait du café et il nous a dit que les choses étaient toujours difficiles entre lui et Jeanne. Les choses, cela veut dire l'amour.» $(B C V, 221)$. 
à «la petite lumière au fond de chacun de nos gestes» $(B C V, 146)$ qui illumine la moindre des choses ${ }^{24}$. Cela n'implique pas un refus de la réalité immédiate et locale. Celle-ci est plutôt la condition de possibilité de l'action et du sens, qui renvoie à un humanisme universel dont la maternité et l'enfance sont exemplaires. Le quotidien n'est autre que cet espace où le local et l'universel se réfléchissent l'un l'autre. Il est alors transfiguré, grandi de l'intérieur, porté vers un avenir certes tremblant, mais possible. Cette possibilité donne au minimalisme intimiste d'Élise Turcotte un élan narratif certain qui, aussi ténu soit-il, le distingue tant des petits bonheurs volés au temps de Philippe Delerm que des dérives de Jean-Philippe Toussaint ou de l'étouffement in vivo de la rencontre amoureuse chez Larry Tremblay 25 .

Contrairement à l'intériorité qui délimite le champ d'une conscience, l'intériorisation est un processus qui comprend mais excède l'espace intime d'une subjectivité inquiète. Dans Le bruit des choses vivantes, elle est aussi une question de récit: les histoires et leurs modalités sont inséparables de la vie ${ }^{26}$. Albanie combat pour mettre de l'ordre dans son existence, établir des liens, et le récit est projeté comme une lutte contre sa propre fragmentation. Non seulement l'existence se lie à travers les images et les fables, mais elle est traversée, structurée, orientée par celles-ci. Ce qui s'intériorise, dans la vie comme dans le récit, par le biais de la narratrice, ce sont les catégories génériques du romanesque et du merveilleux véhiculées par différents médias (télévision, cinéma, littérature) et le personnage de l'enfant. Le romanesque et le merveilleux pénètrent ainsi le cœur des choses, les font parler. Nul besoin, dès lors, d'aventures extraordinaires et de personnages hors du commun pour donner au récit un souffle romanesque : l'extraordinaire pénètre l'ordinaire du monde, il l'anime de l'intérieur, mobilisant du dedans et la "petite vie» et le récit en mode mineur. Ce mode minimaliste tient à la fois au minutieux travail des motifs susceptible de graduellement faire émerger une durée narrative, au symbolisme reliant l'infiniment petit à l'infiniment grand, à l'intrusion d'un incident minuscule perçu comme un événement majeur (lequel entraîne l'action et la finalité à petite échelle dans le roman), et à l'intériorisation de catégories génériques qui, loin des détournements parodiques de ceux qu'on a appelés les nouveaux auteurs de Minuit, donne au récit une profondeur existentielle sans qu'il faille pour autant aller au bout du monde ${ }^{27}$.

L'univers quotidien renvoie à une réalité invisible hantée d'imaginaires, non pas occulte mais idéale, d'ordre métaphysique et psychologique (vie, mort, amour,

$$
+++
$$

24 Le processus d'intériorisation va jusqu'à définir les personnages et leurs relations: alors qu'avec Alexandre, «[t]ous les mots, tous les gestes sont des mouvements de l'extérieur.» (BCV, 46), Pierre, "[1] ui, regarde à l'intérieur. » $(B C V, 184)$. 25 Je pense ici à Anna à la lettre c (Montréal, Les Herbes rouges, 1992). Cette fiction minimaliste et intimiste raconte de l'intérieur, par le menu, une rencontre entre un homme âgé et une jeune fille. Le désir palpable qui s'installe entre eux, porteur de tous les possibles, se solde par un «silence, qui ne peut être rien d'autre que le gonflement du sale, du triste, de l'échoué» (p. 53). 26 On retrouve ce trait chez Annie Ernaux, mais dans une veine hyperréaliste qui s'en tient, autant que possible, à la surface du vu et de l'entendu. 27 En ce sens, le fait que Le bruit des choses vivantes se suspende juste avant le grand voyage d'Albanie et de Maria est significatif. Précisons que le «rêve de voyage» dans le Nord n'est que plus absolu, plus idéal, de n'être pas raconté. 
abandon, etc.) ${ }^{28}$. Cet alliage de quête existentielle et d'imaginaire romanesque fait de l'intériorisation une force de révélation. Mais en renouant avec la puissance de figuration d'un quotidien intériorisé, Turcotte dématérialise le monde matériel. La moindre des choses acquiert une profondeur de sens et une légèreté remarquables. Dans l'univers d'Albanie, les piles de linge veulent dire symboliquement beaucoup (désordre, nécessité de mettre de l'ordre) mais ne posent aucun problème concret. Nul penchant, comme chez Jean-Philippe Toussaint, à s'appesantir sur les microscopiques mésaventures du quotidien pour faire ressortir l'épuisante résistance de la réalité. Nul besoin non plus de souligner l'aliénation quotidienne qui gouverne nos vies, à la manière d'Annie Ernaux dans un registre hyperréaliste au féminin ${ }^{29}$. La maternité est légère chez Turcotte: il n'est jamais question de corvée, de galère. Même la fatigue semble flotter: c'est l'idée qui compte, ce que «ça veut dire». Les images et les histoires conjurent l'insignifiance du quotidien, elles permettent de relier les instants, les êtres, le monde et le roman lui-même. La vie de tous les jours renvoie ainsi à des questions universelles plutôt qu'à une réalité contingente ou routinière. Les gestes et les mots illuminent les êtres, l'univers est une forêt de motifs et de symboles entrelacés, un kaléidoscope d'images à mettre en boîte et en récit. Nous franchissons le pas: à l'intérieur du quotidien, il y a le quotidien révélé.

$$
++
$$

28 L'idéalité implique non pas l'évacuation du tragique, mais la capacité à refléter, dans les bonheurs et les malheurs singuliers, l'idée du Bonheur et du Malheur, du Bien et du Mal, de la Vie et de la Mort. 29 «Je n'ai plus connu qu'un temps uniformément encombré d'occupations hétéroclites. Le linge à trier pour la laverie, un bouton de chemise à recoudre, rendez-vous chez le pédiatre, il n'y a plus de sucre. L'inventaire qui n'a jamais ému ni fait rire personne. Sisyphe et son rocher qu'il remonte sans cesse, ça au moins quelle gueule, un homme sur une montagne qui se découpe dans le ciel, une femme dans sa cuisine jetant trois cent soixante-cinq fois par an du beurre dans la poêle, ni beau ni absurde, la vie Julie. » Annie Ernaux, La femme gelée, Paris, Gallimard, coll. «Folio», 2003 [1981], p. 155. 